\title{
OTIOTOMTCS
}

Revista de economía, empresa y sociedad

RETO DE FUTURO

\section{Blockchain y las criptomonedas: el caso bitcoin}

\section{José Miguel Domínguez Jurado}

Profesor titular de Economía Aplicada (Universidad de Cádiz)

\section{Ricardo García Ruiz}

Profesor colaborador del grado de Información y Documentación (UOC)

RESUMEN El objetivo de este artículo es explicar cómo un proceso informático, la blockchain o 'cadena de bloques', permite generar la confianza necesaria para la creación, la aceptación y el uso cada vez más generalizado de las monedas digitales, bajo un nuevo concepto o paradigma de dinero no sostenido por ningún ente estatal o supranacional: las criptomonedas. Para conocer este proceso, en primer lugar se exponen los aspectos técnicos de funcionamiento de la cadena de bloques y de cómo esta ha conseguido generar confianza en el usuario. En segundo lugar, se analiza su implementación en la más conocida de todas, bitcoin, así como los datos relacionados con la expansión y el uso de las principales criptomonedas, en un mercado que en los últimos dos años se ha multiplicado por 65, alcanzando el medio billón de dólares. El trabajo se cierra exponiendo las principales conclusiones y reflexiones.

PALABRAS CLAVE bitcoin, divisas digitales, mercado de divisas, blockchain, sistema de pagos 


\title{
«Blockchain» and cryptocurrencies: Bitcoin
}

\begin{abstract}
The main aim of this article is to explain how a computer process, the "Blockchain» or chain of blocks, can generate the necessary trust for the creation, acceptance and increasingly widespread use of digital currencies, under a new concept or paradigm of money not maintained by any state or supranational entity: cryptocurrencies. In order to understand this process, the technical aspects of how the blockchain operates and how it has managed to generate confidence in the end user are explained first. Secondly, its implementation will be analyzed through the best known of all, Bitcoin, and through the data related to the expansion and use of the main cryptocurrencies in a market that in the last two years has multiplied by 65, reaching half a trillion dollars. This work closes by exposing the main conclusions and reflections.
\end{abstract}

KEYWORDS bitcoin; digital currency; currency market; blockchain; payment system

\section{Introducción}

El desarrollo tecnológico ha contribuido de manera decisiva a la transformación de la economía y al progreso de la humanidad. Si el hito más importante fue la propia Revolución Industrial, hoy día estamos asistiendo a otra revolución cuyo origen ha sido la invención de los ordenadores, el desarrollo de software, la interconexión a escala mundial y su uso masivo desde principios del siglo xxi. Esto ha aumentado las posibilidades de crear nuevos productos y servicios, y ha abierto la puerta a transformaciones de gran calado que incluso permitan hablar de una nueva economía que conviva con la actual, o que incluso en un futuro pueda llegar a sustituirla.

Entre esas innovaciones está la blockchain, una herramienta desarrollada dentro del campo de la criptografía y la gestión de redes informáticas. Blockchain es un sistema tecnológico que tiene entre sus objetivos principales generar confianza a sus usuarios, y la forma en la que está concebido impide, al menos a priori, ejercer ningún control por parte de usuarios u organizaciones, lo que está significando una revolución.

El proceso más conocido basado en la blockchain, y en rápida expansión, es el de emisión de una moneda virtual, denominada como «criptomoneda», y que ha dado lugar al crecimiento del mercado de criptomonedas en su número, en valor de capitalización y en volumen de transacciones. Es un comportamiento que en 
los últimos meses podría calificarse de explosivo, a tenor de su intenso crecimiento; incluso la propia banca tradicional está operando con criptomonedas (Rivero, 2017; Leal, 2017).

Pero su papel no queda ahí, sino que las posibilidades de aplicación de la blockchain en otras parcelas de la economía y el derecho pueden ser innumerables, ya que esta estructura de archivos es muy adecuada para asegurar escritos de valor crítico, grabaciones producidas de manera continua y que tengan valor legal, tales como operaciones de pago, pero también muchos otros tipos de escritura como títulos de propiedad, patentes, diplomas, etc. (Ammous, 2016; Pilkington, 2015).

Partiendo de la definición y de los aspectos técnicos del funcionamiento de la blockchain, es fácil comprender la confianza que genera en sus usuarios. Además, el hecho de no depender de una institución u organización que la respalde, dota a los procesos que utilizan esta tecnología de gran libertad, tanto en su creación como en su implementación, por no decir que, en muchos casos, el hecho de poder eludir el control se convierte en el leitmotiv de algunos productos.

Sin embargo, un proceso tan novedoso no está exento de problemas, por lo que se hace necesario efectuar una reflexión sobre las perspectivas de futuro de la blockchain aplicada a otros campos en general, y las criptomonedas en particular.

\section{Fundamentos de la cadena de bloques (blockchain)}

Blockchain es una base de datos de creación colectiva, cuyos componentes históricos no pueden ser modificados. Se crea, se difunde y se accede a la misma a través de una red distribuida sin nodos privilegiados.

Esta definición esconde un proceso de funcionamiento y creación complejo, que otorga las garantías de seguridad necesarias para que las acciones que se lleven a cabo sean confiables y aceptadas por sus usuarios. Sus ventajas son evidentes, especialmente en integridad de los datos y certificación. Nadie puede cambiar la información almacenada en un bloque blockchain (Lemieux, 2016), y se puede aplicar en el ámbito de acreditación y seguridad jurídica.

El proceso de construcción de una blockchain garantiza su seguridad, integridad e independencia. Además, genera empleo: los «mineros», que se encargan de las operaciones necesarias de creación y consolidación de bloques, y obtienen una remuneración como compensación.

La blockchain se define como «una base de datos distribuida». Destacan las características siguientes: 
- Opera de manera descentralizada, P2P, ${ }^{1}$ sin supervisión por parte de ninguna autoridad central (Swan, 2015). Cada nodo tiene una copia propia de la blockchain y contribuye en la construcción y el mantenimiento de las operaciones.

- La base de datos solo admite adiciones en orden cronológico; es imposible cambiar o borrar información ya registrada.

- Es un registro certificado criptográficamente, asegurando la consistencia de todos los datos registrados o almacenados, y validados por la mayoría de los nodos de la red (Nakamoto, 2008). Esta característica permite que la blockchain sirva de certificación de integridad para cualquier transacción.

La blockchain está formada por bloques de información. En el caso de las criptomonedas, son mayoritariamente las transacciones monetarias válidas. Los bloques se enlazan unívocamente en una cadena. Cada bloque incluirá un $\boldsymbol{h a s h}^{2}$ del bloque anterior, encadenándose con el anterior, manteniendo la consistencia y unicidad.

\section{Gráfico 1. Funcionamiento de la blockchain}

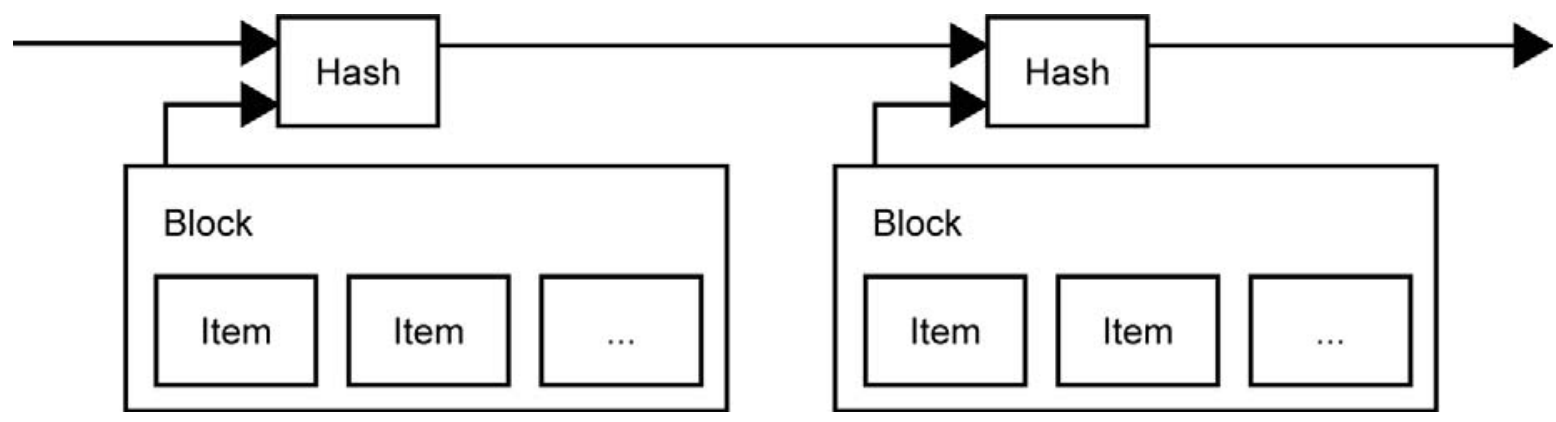

Fuente: Nakamoto (2008).

Un bloque está formado por unos registros de datos, cuya forma y significado dependen de su aplicación. Para bitcoin y otros sistemas de pago, estas entradas son las transacciones monetarias: cada bloque contiene entre 1.000 y 3.000 . Un bloque contiene un encabezado con su suma de control, un identificador del bloque anterior (con su propia suma de comprobación). Se puede verificar cualquier bloque navegando hasta el bloque inicial de la cadena. La suma de comprobación de un bloque siempre se comprueba en cada acceso, para detectar cualquier

1. P2P es el acrónimo de peer to peer, o red entre pares; las computadoras se conectan y comunican entre sí sin servidor central, aprovechando, optimizando y administrando el ancho de banda de la red.

2. Una suma de comprobación (hash) es un número, calculado a partir del contenido del bloque. Cualquier modificación intencional de cualquier bloque cambia la suma de comprobación de modo impredecible. Dos bloques distintos siempre tienen diferentes hash. 
cambio en su contenido tras su construcción. Una modificación del bloque, incluyendo la cabecera, con su nueva suma de comprobación, implica modificar su identificador (Stahr, 2014).

\subsection{Construcción de una cadena de bloques}

El proceso de creación es competitivo y abierto: esto le confiere su independencia. La competencia se basa en quién logra el cierre del bloque, por parte de los mineros, ya que solo el primero que cierre el bloque de manera correcta y validada será remunerado. Es abierto por la estructura de trabajo en red.

La verdadera originalidad del bitcoin y criptomonedas derivadas, que les dan sus cualidades únicas y su complejidad, es que se ubican en una red peer to peer abierta, sin servidor central ni nodos privilegiados. Cualquier persona puede unirse a la red sin tener que pedir permiso. El software utilizado es de libre acceso. El usuario puede descargar, instalar y ejecutar este software sin necesitar autorización. Aunque hay una plataforma preferida de distribución (Github) y software recomendado por distintas instancias (Bitcoin.org, 2011; Hartikka, 2016; ethereum.org, 2018), cualquier persona puede proponer un nuevo software o usar una plataforma diferente (Tormey, 2017).

El proceso de generación del blockchain se articula en tres ámbitos:

- Difundir la escritura entre nodos: las operaciones son visibles para todos los operadores.

- Construcción de los bloques.

- Construcción de la cadena.

Como primer paso, cada nueva creación de un nodo entra en el sistema por un nodo de la red, verificando que su estructura es correcta y válida de acuerdo con las entradas ya grabadas. En el caso de bitcoin, se verifica por transacciones anteriores que hay en la cuenta una cantidad al menos igual a la suma para pagar, y que el autor de la transacción está autorizado. Si se cumplen estas condiciones, la escritura del nodo queda en espera en una lista local y distribuida a toda la red. De lo contrario, se rechaza.

Cada tipo de anotación requiere su protocolo de validación, que define las entradas válidas en el sistema. Este protocolo se ejecuta sucesivamente en las etapas de construcción y validación de los bloques. Cada sistema puede contener un directorio de protocolos de validación, cada uno asociado con un código en la parte superior de cada secuencia de comandos, lo que permite llevar a cabo anotaciones en la misma cadena de bloques sobre transacciones de distintos tipos. 
Al recibir cada mensaje, se comprueba la validez formal y la legitimidad sobre su información. La naturaleza abierta de la red impone un principio fundamental de seguridad: cada nodo asume que el resto del sistema puede ser defectuoso o fraudulento. Todo el sistema está diseñado robustamente bajo la suposición de que está constantemente bajo ataque por defraudadores altamente cualificados.

Tras esto, las entradas no válidas se han eliminado, y cada anotación válida y legítima se ha copiado en la red distribuida, siendo debidamente validada en cada etapa de su trayectoria desde su punto de entrada.

En la segunda etapa, los mineros colocan las entradas en su lista de espera local y las organizan con el fin de facilitar el acceso a las entradas individuales. Todo minero trabaja de manera simultánea y elige libremente qué registros incorpora en cada bloque en construcción. Se termina el bloque añadiendo una cabecera con la suma de comprobación y el identificador del bloque anterior.

El coste computacional de esta operativa es intencionadamente muy alto. La suma de comprobación cumple normas muy estrictas que requieren muchos recursos informáticos, pero cuya verificación pueda ser comprobada fácilmente por todos los nodos: se llama «prueba de trabajo». Este requisito limita el minado individual (grupos en modo cooperativo), ya que para que sus bloques sean aceptados, necesitan mucha capacidad de cálculo (IBM Bluemix, 2017).

Existe remuneración como motivación al minado. En bitcoin, esta se hace automáticamente mediante la inserción del bloque en una transacción especial, acreditando al minero un número de bitcoins ya creados para este propósito, cuando el bloque es añadido finalmente. Si un minero ha construido un bloque válido, lo transmite a sus vecinos, y estos a su vez lo retransmiten a todos los nodos de la red, tras comprobar la validez de todas sus entradas. Cuando un minero recibe un nuevo bloque, cesa de construir su bloque actual, ya que no se aceptará, y comienza a construir otro nuevo bloque.

La construcción de la cadena. Para cada nuevo bloque recibido, cada ordenador de un nodo completo ejecuta un protocolo establecido, ya sea para rechazar este bloque, porque no sea válido o presente ese contenido en la escritura local, añadirlo al final de la cadena local, después de una comprobación final de todas las entradas en el mismo, o ponerlo en espera. Cada bloque contiene el identificador de su bloque antecesor en la blockchain del minero que la construyó. El predecesor es el bloque terminal de la cadena local, y se añade tras una verificación final de su validez. De lo contrario, será retenido en cola y será el extremo de una rama secundaria de la blockchain (Crosby, 2016; Ateniese y otros, 2017).

Unicidad y consistencia. El protocolo incorpora un mecanismo de consenso como parte vital del sistema. La regla en bitcoin es mantener la rama con mayor trabajo de construcción. En la cabecera de cada bloque, hay un valor numérico del trabajo efectuado para su construcción: la suma de este valor para cada rama 
indica el trabajo conjunto llevado a cabo. Si la suma del valor de una rama secundaria es superior al valor de la suma de la rama principal actual, entonces esa rama secundaria se convertirá en la nueva rama principal. El software llevará a cabo los ajustes y validaciones de bloques necesarios. Este protocolo crítico y complejo es el verdadero corazón del sistema.

Todas las copias de la blockchain se mantendrán idénticas, ya que se aplican las mismas reglas de validación de entradas y bloques.

Todos los millones de entradas creadas se encuentran registradas en miles de archivos idénticos en sitios diferentes, inalterables, y pueden consultarse de manera gratuita (IBM Bluemix, 2017; Kehrli, 2016).

Gráfico 2. Diferencias gráficas entre sistemas de redes

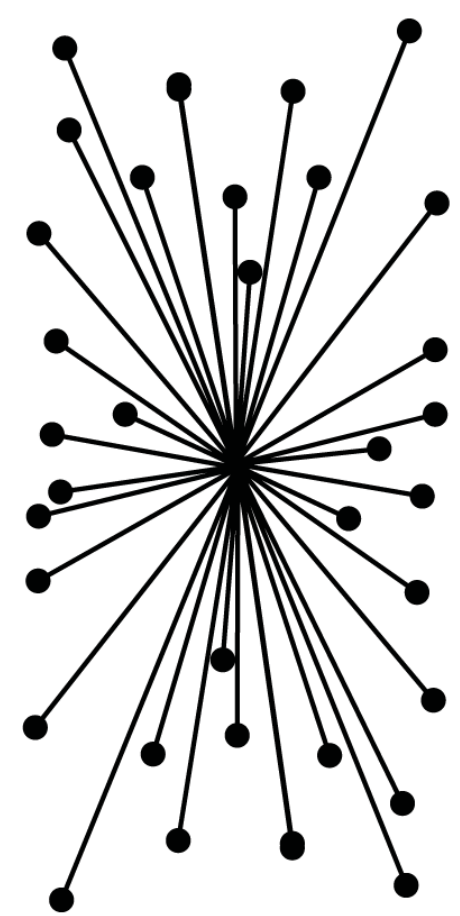

Centralizada

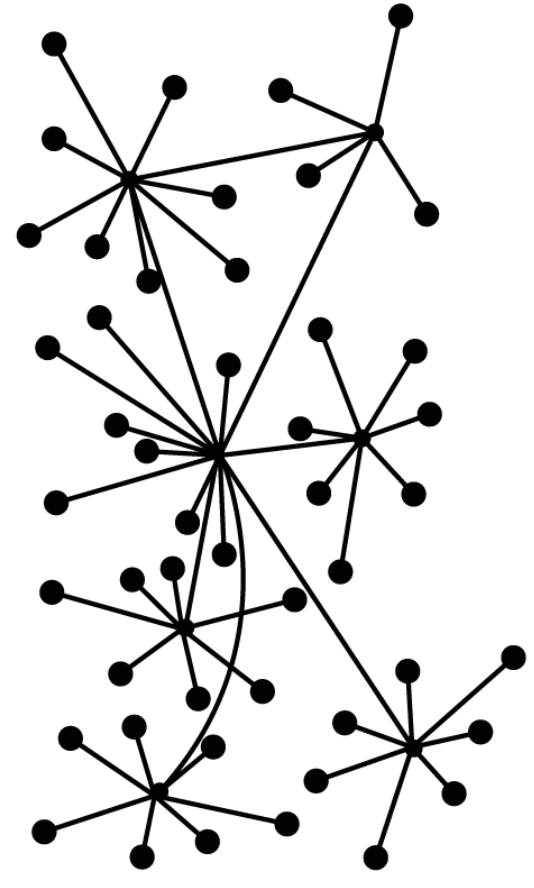

Descentralizada

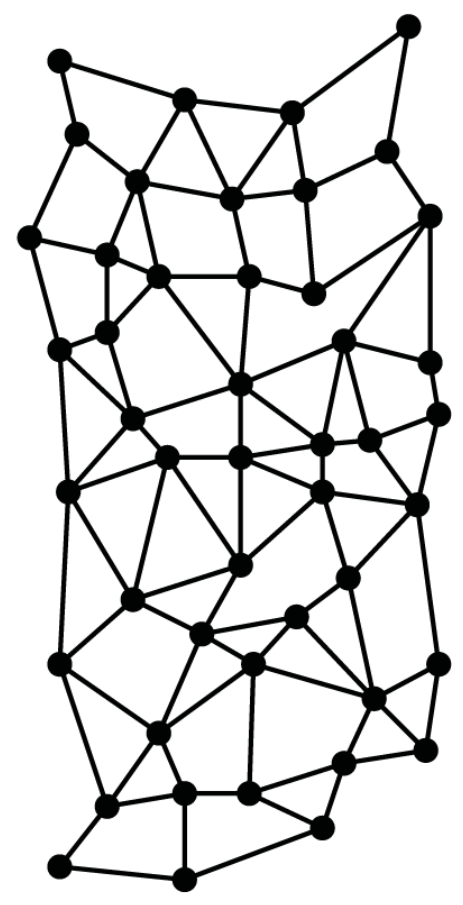

Distribuida

Fuente: Las tres topologías de red según los grafos de Paul Baran.

\section{Las criptomonedas: el caso del bitcoin}

En la actualidad, bitcoin es la más conocida y utilizada de las muchas criptomonedas que existen hoy día. Antes de la aparición de bitcoin, existieron precursores de monedas electrónicas, como por ejemplo las tecnologías desarrolladas por David Chaum y Stefan Brands (moneda electrónica), Adam Back (hashcash), Wei Dai 
(b-dinero) o Nick Szabo (bit-oro) (Brands y Chaum, 1993; Chaum y Brands, 1997; Back, 2002).

El mercado de criptomonedas, hasta fechas muy recientes, ha estado prácticamente monopolizado por el bitcoin. Hablar de capitalización del mercado era sinónimo de hablar de capitalización del bitcoin. El gráfico 3 muestra la evolución de su valor desde su primera cotización hasta la actualidad.

\section{Gráfico 3. Capitalización del mercado de bitcoins}

\section{Millones de dólares}

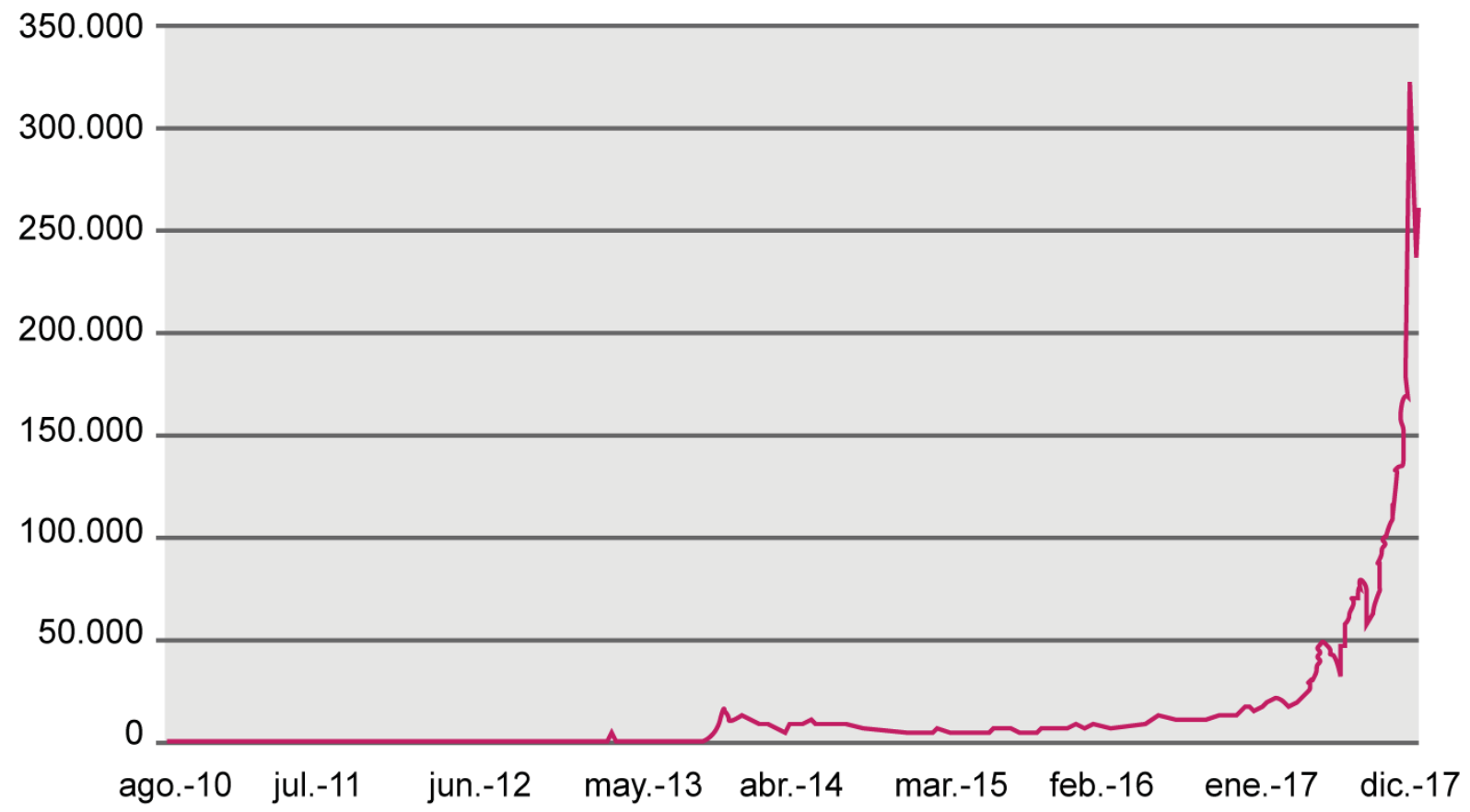

Fuente: Elaboración propia a partir de blockchain.info.

Como elementos más destacables, hay que reseñar que el 2013 marca el despegue del bitcoin, y se inicia un periodo de expansión de su capitalización hasta diciembre del 2014, cuando alcanza los 14.000 millones de dólares. Tras este máximo, su valor desciende paulatinamente durante el 2014, especialmente en diciembre y enero del 2015, y llega a perder más del 80 \% de su valor de capitalización. Este toque de atención estabilizó el mercado durante todo el 2015, advirtiéndose notas de recuperación a finales de año, en torno a los 6.500 millones.

El año 2016 muestras signos de fortaleza, e incrementa progresivamente el valor de capitalización, lo que a mitad de este año permite recuperar el nivel del 2014, cerrando con un volumen de capitalización cercano a los 15.000 millones, e iniciándose un proceso explosivo a partir de abril del 2017. 
Gráfico 4. Capitalización del mercado de criptomonedas y bitcoins

Millones de dólares

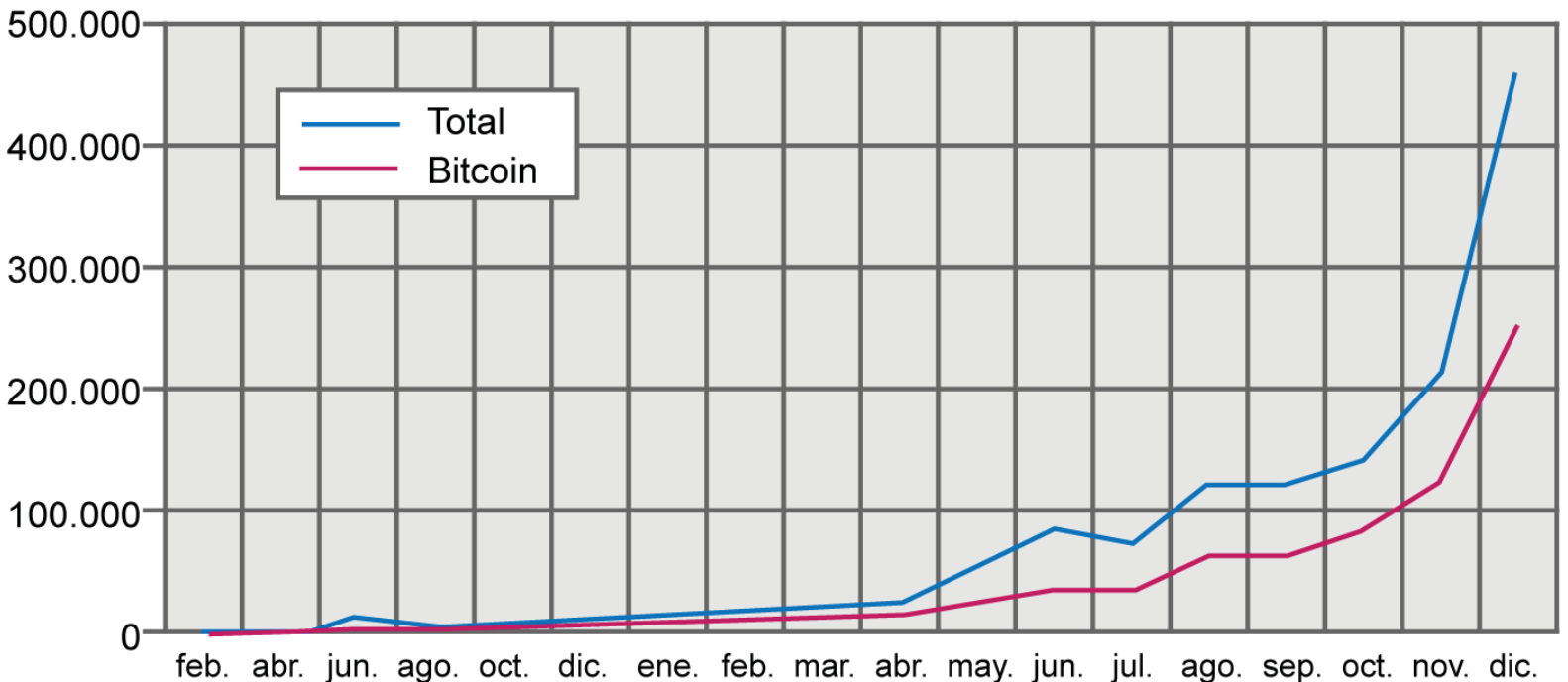

2016

2017

Fuente: Elaboración propia a partir de coingecko.com

El gráfico 4 presenta la evolución del valor medio mensual de la capitalización del mercado total y del bitcoin. Podemos destacar tres fases: la primera, de lenta expansión del mercado hasta abril del 2017, condicionada por el monopolio de bitcoin. A partir de esta, fecha, se inicia una segunda etapa con un notable incremento de la tendencia y, por ende, del crecimiento del mercado, aunque con una evolución irregular, y destaca una pérdida de importancia relativa del bitcoin por el nacimiento y la expansión de otras criptomonedas. A partir de septiembre del 2017, se inicia una tercera fase caracterizada por un crecimiento explosivo, prácticamente exponencial, pero se advierte claramente que el peso relativo del bitcoin desciende, motivado por el fuerte crecimiento de otras criptomonedas que están ganando cuota de mercado, y se detectan grandes irregularidades, debido a fuertes movimientos especulativos.

Tabla 1. Capitalización del mercado (\% de media mensual)

\begin{tabular}{|c|c|c|c|c|c|c|c|}
\hline & Bitcoin & Etherum & Ripple & $\begin{array}{c}\text { Bitcoin } \\
\text { Cash }\end{array}$ & Cardano & Litecoin & Resto \\
\hline enero & $89,22 \%$ & $5,39 \%$ & $1,45 \%$ & & & $1,21 \%$ & $2,73 \%$ \\
\hline febrero & $89,40 \%$ & $5,67 \%$ & $1,21 \%$ & & & $1,03 \%$ & $2,70 \%$ \\
\hline marzo & $80,01 \%$ & $13,21 \%$ & $1,20 \%$ & & & $0,89 \%$ & $4,70 \%$ \\
\hline abril & $71,64 \%$ & $16,57 \%$ & $4,71 \%$ & & & $2,01 \%$ & $5,07 \%$ \\
\hline
\end{tabular}




\begin{tabular}{|c|l|l|l|l|l|l|l|}
\hline & Bitcoin & Etherum & Ripple & $\begin{array}{c}\text { Bitcoin } \\
\text { Cash }\end{array}$ & Cardano & Litecoin & Resto \\
\hline mayo & $55,42 \%$ & $20,15 \%$ & $15,13 \%$ & & & $2,44 \%$ & $6,85 \%$ \\
\hline junio & $47,05 \%$ & $31,45 \%$ & $11,95 \%$ & & & $2,06 \%$ & $7,50 \%$ \\
\hline julio & $52,00 \%$ & $26,70 \%$ & $9,85 \%$ & & & $2,93 \%$ & $8,51 \%$ \\
\hline agosto & $52,75 \%$ & $23,28 \%$ & $6,02 \%$ & $6,35 \%$ & & $2,13 \%$ & $9,47 \%$ \\
\hline septiembre & $53,38 \%$ & $22,24 \%$ & $6,07 \%$ & $6,57 \%$ & $0,00 \%$ & $2,50 \%$ & $9,24 \%$ \\
\hline octubre & $60,11 \%$ & $19,90 \%$ & $5,93 \%$ & $4,06 \%$ & $0,46 \%$ & $2,05 \%$ & $7,50 \%$ \\
\hline noviembre & $61,17 \%$ & $16,18 \%$ & $4,13 \%$ & $8,67 \%$ & $0,40 \%$ & $1,74 \%$ & $7,70 \%$ \\
\hline diciembre & $56,26 \%$ & $13,46 \%$ & $6,43 \%$ & $7,94 \%$ & $1,70 \%$ & $2,71 \%$ & $11,51 \%$ \\
\hline 31/12/17 & $43,60 \%$ & $13,88 \%$ & $16,36 \%$ & $8,09 \%$ & $3,36 \%$ & $2,39 \%$ & $12,34 \%$ \\
\hline
\end{tabular}

Fuente: Elaboración propia a partir de coingecko.com.

Así pues, aunque el bitcoin es el que marca la fortaleza y evolución del mercado, la creación y expansión de otras criptomonedas han reducido su importancia en el conjunto, con un descenso continuado desde marzo del 2017, que lo llevó en junio hasta el 52,00 \% de la cuota del mercado, y se recuperó a finales de año hasta el $56,26 \%$ (tabla 1). A este hecho se le une la escisión del bitcoin en tres criptomonedas: bitcoin, bitcoin cash y bitcoin gold.

Con todo esto, bitcoin cierra el año (31 de diciembre del 2017) con una posición de dominio, con el 43,62 \% de cuota de mercado, a la que sigue, en orden de importancia, ripple con el $16,36 \%$, ethereum, con el $13,88 \%$, y dos monedas de nueva creación: bitcoin cash, con el 8,09 \% y cardano, con el 3,36, \%, cuotas conseguidas en el corto espacio de tiempo de 5 y 3 meses, respectivamente, desde su creación.

Las particularidades del bitcoin frente a las monedas tradicionales y otras criptomonedas se pueden resumir en los siguientes puntos:

- No necesita un banco central: es una moneda electrónica, descentralizada, supuestamente anónima, no apoyada por ningún Gobierno o entidad legal. Las comprobaciones son llevadas a cabo por usuarios de la red Bitcoin.

- El proceso de emisión es automático, predecible y no está al servicio de ningún usuario privilegiado.

- El valor de un bitcoin es determinado por el mercado de divisas mediante su uso.

- No se basa en bienes materiales, sino en el valor que los individuos le otorgan.

El proceso de crecimiento de bitcoin limitará su oferta a unos 21 millones, lo que unido a su demanda creciente hace que su valor se haya disparado en los últimos 
meses, y su cotización a 31 de diciembre del 2017 es de aproximadamente 14.000 dólares por bitcoin, llegando casi a los 20.000 dólares durante algunos días de este mes. El gráfico 5 muestra la evolución temporal del valor del bitcoin. Así pues, la oferta de bitcoins no se ajusta a la riqueza intercambiada a través del mismo, sino que estaría más cerca del concepto de metales preciosos como el oro, lo que llevó a algunos a definir el bitcoin como el oro digital (Popper, 2016).

Gráfico 5. Bitcoins: valor de mercado y número

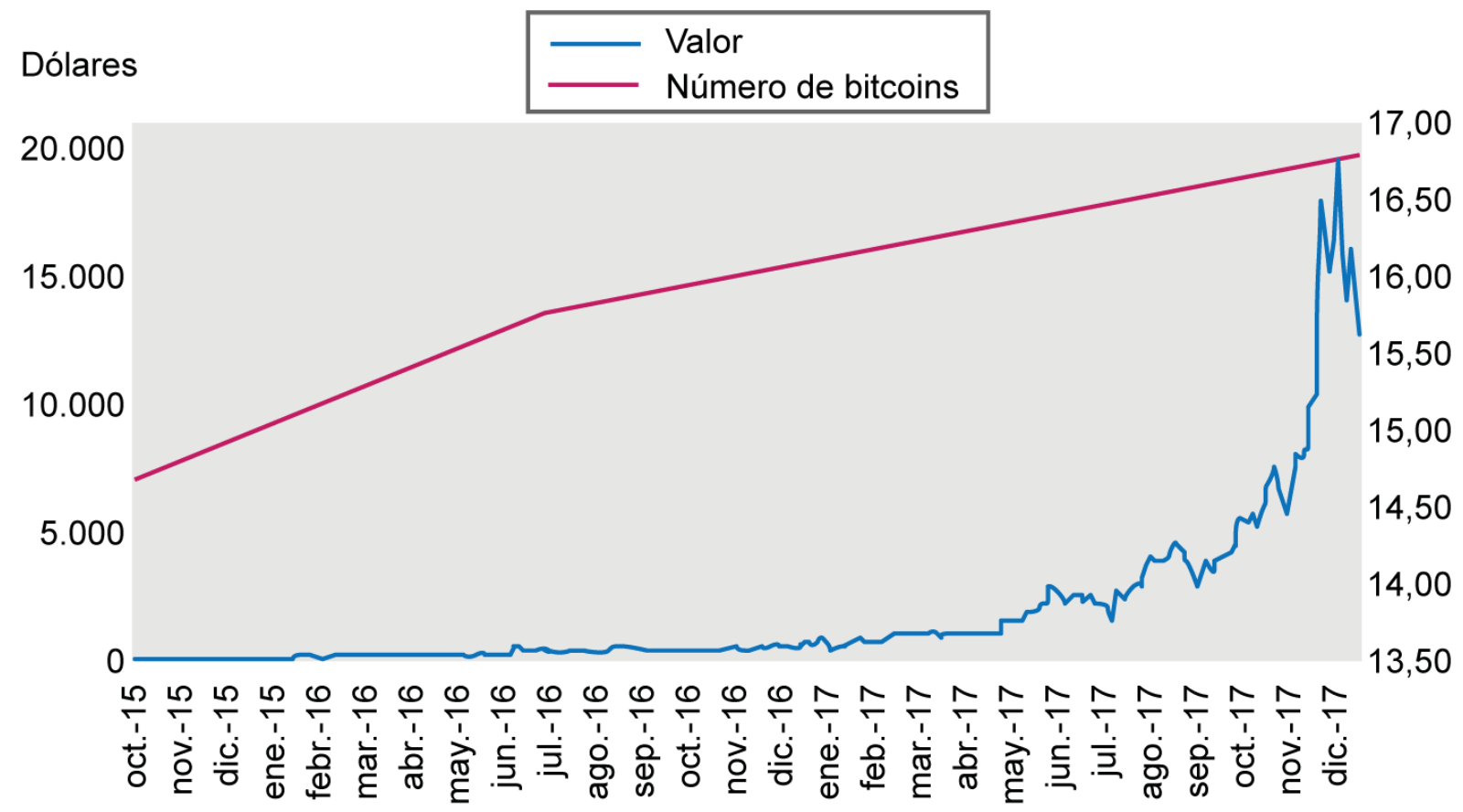

Fuente: Elaboración propia a partir de acoingecko.com.

\section{Conclusiones y reflexiones finales}

Podríamos definir blockchain como una tecnología informática en la que un conjunto de actores registra las transacciones efectuadas entre usuarios y sobre la que, al ser una red descentralizada, nadie puede a priori ejercer ningún tipo de control, y ofrece la confianza necesaria para la aceptación de una criptomoneda. Además de ser el apoyo de procesos económicos y legales, la blockchain está creando nuevas profesiones (mineros) y actividades económicamente remunerables con criptomonedas.

El ejemplo más conocido y referente del mercado de criptomonedas ha sido el bitcoin que, tras un periodo de incertidumbre y dudas tras su creación, a partir del 2013 ha iniciado una senda expansiva; junto con el bitcoin, han aparecido nuevas monedas con un intenso crecimiento de su capitalización, lo que ha hecho que el bitcoin pierda peso relativo en el conjunto. 
El año 2017 ha sido crucial en el crecimiento y la consolidación de este mercado, que ha expandido el valor de capitalización al medio billón de dólares, dominado por el bitcoin, que representa prácticamente la mitad del mercado. Sin embargo, tras este fuerte crecimiento se advierten fuertes procesos especulativos.

El bitcoin está programado para llegar a un límite de unidades en torno a los 21 millones, lo que condiciona su precio al alza en los mercados, valor que en los últimos tres meses del 2017 se ha multiplicado por 4, llegando a alcanzar casi 20.000 dólares, por lo que ha sido necesario fraccionar la moneda en unidades menores de intercambio (satoshi).

Por lo tanto, cabe preguntarse si las criptomonedas están en condiciones de reemplazar, al menos a priori, al sistema de moneda tradicional basada en la participación de un tercero (banco o banco central) capaz de asumir una función de la regulación (Sandoval, 2017; ethereum.org, 2018). Algunos estados, como es el caso de Dinamarca, ya plantean la retirada de la moneda tradicional y adoptar este tipo de monedas, pero bajo su supervisión.

Los dos grandes problemas a los que se enfrenta bitcoin son la colusión de los mineros y la especulación. Si un grupo mayoritario de mineros se pusiesen de acuerdo, convertiría a la red en lo que podría denominarse un «banco central privado», cosa poco probable, pero posible. Los riesgos de la especulación son evidentes, y derivan precisamente de no disponer de una producción real que respalde la criptomoneda: tal y como ha aparecido, podría desaparecer. El ciudadano no especializado ya se está interesando de manera creciente con este último aumento de precios, quizá más con fines especulativos que de uso en sí de esta moneda como mecanismo de pagos (Arce, Martínez, 2016; Asensio Grau, 2014; Elwell, Murphy y Seitzinger, 2013; Gallén, 2016; Grinberg, 2011; Gutiérrez Hernández, 2015; Europa Press, 2016; Unidad Editorial Información Económica, 2017).

A modo de apunte final, no debemos olvidar que la forma de trabajo de la blockchain trasciende a otros campos económicos y del derecho, ya que tienen el potencial de cambiar de manera fundamental el tejido mismo de nuestra sociedad en un ámbito global. Puede hacer esto dando a miles de millones de personas el acceso a un sistema financiero global, y además, en la aplicación de otros muchos usos no financieros aún no conocidos, pero que están viendo la luz en este mismo momento (Popper, 2016).

\section{Referencias bibliográficas}

AMMOUS, S. H. (2016). «Blockchain Technology: What is it Good for?» [en línea]. [Consulta: 20 de mayo del 2017] <https://papers.ssrn.com/sol3/papers. cfm?abstract_id=2832751> 
ARCE, D. (2016). «Transformación de la forma tradicional de banca hacia el mundo digital» [en línea]. [Consulta: 7 de julio del 2017] <http://repositorio.upct.es/ handle/10317/5900>

ASENSIO, Y. (2014). «Bitcoin: La nueva moneda virtual que está revolucionando el mundo de las divisas digitales» [en línea]. [Consulta: 7 de julio del 2017] <http:// repositori.uji.es/xmlui/handle/10234/112560>

ATENIESE, G.; MAGRI, B.; VENTURI, D.; ANDRADE, E. (2017). «Redactable Blockchain -or- Rewriting History in Bitcoin and Friends» [en línea]. [Consulta: 25 de junio del 2017] <http://eprint.iacr.org/2016/757.pdf>

BACK, A. (2002). "Hashcash - A Denial of Service Counter-Measure» [enlínea]. [Consulta: 25 de junio del 2017] <http://citeseer.ist.psu.edu/viewdoc/ summary?doi=10.1.1.15.8>

BITCOIN.ORG (2011). «Bitcoin.org's Codebase» [en línea]. <https://github.com/ Bitcoin-dot-org/Bitcoin.org>

BRANDS, S.; CHAUM, D. (1993). «Distance-Bounding Protocols». Advances in Cryptology - EUROCRYPT '93 (págs. 344-359). Berlín, Heidelberg: Springer Berlin Heidelberg [en línea]. [Consulta: 25 de junio del 2017] <http://link.springer.com/10.1007/3-540-48285-7_30>

CHAUM, D.; BRANDS, S. (1997). «Minting» electronic cash». IEEE Spectrum (vol. 34, núm. 2, págs. 30-34) [en línea]. [Consulta: 25 de junio del 2017] ISSN 00189235. DOI 10.1109/6.570825. <http://ieeexplore.ieee.org/document/570825/> CROSBY, M. (2016). «BlockChain Technology: Beyond Bitcoin. Applied Innovation Review (núm. 2) [en línea]. [Consulta: 25 de junio del 2017] <http://scet.berkeley.edu/wp-content/uploads/AIR-2016-Blockchain.pdf>

ELWELL, C. K.; MURPHY, M. M.; SEITZINGER, M. V. (2013). «Bitcoin: Questions, Answers, and Analysis of Legal Issues». Congressional Research Service. Informing the legislative debate since 1914 [en línea]. [Consulta: 7 de julio del 2017] $<$ http://www.a51.nl/storage/pdf/R43339.pdf>

ETHEREUM.ORG (2018). «Ethereum» [en línea]. <https://github.com/ethereum> EUROPA PRESS (2016). "Santander y otros grandes bancos lanzan "su Bitcoin" utilizando la tenología blockchain". elEconomista.es [en línea]. [Consulta: 7 de julio del 2017] <http://www.eleconomista.es/empresas-finanzas/noticias/7782103/08/16/Economia-Santander-se-une-a-cinco-entidades-parapromover-el-uso-de-dinero-digital-entre-entidades-financieras.html>

GALLÉN, P. (2016). «¿Por qué están creando los bancos su propio ‘Bitcoin’?». El Mundo [en línea]. [Consulta: 7 de julio del 2017] <http://www.elmundo.es/econ omia/2016/08/24/57bdc58746163fca1b8b457c.html>

GRINBERG, R. (2011). «Bitcoin: An Innovative Alternative Digital Currency». Hastings Science \& Technology Law Journal (vol. 4, pág. 160) [en línea]. [consulta: 7 de julio del 2017] <https://papers.ssrn.com/sol3/papers.cfm?abstract_id=1817857> 
GUTIÉRREZ, P. (2015). «El Bitcoin ¿presente y futuro del dinero? : sus características e implicaciones» [en línea]. [Consulta: 7 de julio del 2017] <https://repositorio.comillas.edu/jspui/handle/11531/4523>

HARTIKKA, L. (2016). "Naivechain - a blockchain implementation in 200 lines of code» [en línea]. <https://github.com/lhartikk/naivechain>

IBM BLUEMIX (2017). «Blockchain basics». IBM Bluemix Docs [en línea]. [Consulta: 25 de junio del 2017] <https://console.bluemix.net/docs/services/blockchain/ ibmblockchain_overview.html>

KEHRLI, J. (2016). «Blockchain Explained». Netguardians [en línea]. [Consulta: 25 de junio del 2017] <https://www.netguardians.ch/news/2016/11/17/blockchain-explained-part-1>

LEAL, A. (2017). «Banco Central Europeo define la blockchain como 'fuerza transformadora' financiera». Criptonoticias.com [en línea]. [Consulta: 3 de julio del 2017] <https://criptonoticias.com/bancos/banco-central-europeo-define-ablockchain-como-fuerza-transformadora-financiera/\#axzz4|kKrIFRL>

LEMIEUX, V. L. (2016). "Trusting records: is Blockchain technology the answer?». Records Management Journal (núm. 2, vol. 26, págs. 110-139) [en línea]. [Consulta: 24 de junio del 2017]. ISSN 0956-5698. DOI 10.1108/RMJ-12-20150042. <http://www.emeraldinsight.com/doi/10.1108/RMJ-12-2015-0042>

NAKAMOTO, S. (2008). «Bitcoin: A Peer-to-Peer Electronic Cash System» (pág. 9) [en línea]. [Consulta: 24 de junio del 2017] <www.Bitcoin.org>

PILKINGTON, M. (2015). «Blockchain Technology: Principles and Applications» [en línea]. [Consulta: 20 de mayo del 2017] <https://papers.ssrn.com/sol3/Papers. cfm?abstract_id=2662660>

POPPER, N. (2016). «Digital Gold: Bitcoin and the Inside Story of the Misfits and Millionaires Trying to Reinvent Money» [en línea]. [Consulta: 13 de diciembre del 2017] <https://hrgllkeyl.updog.co/aHJnbGxrZXIsMDA2MjM2MjUwWA. pdf $>$

RIVERO, J. (2017). «Banco Central de China abre Centro de Investigación de Monedas Digitales». Criptonoticias.com [en línea]. [Consulta: 3 de julio del 2017] $<$ https://criptonoticias.com/bancos/banco-popular-china-centro-investigacion-monedas-digitales/\#axzz4|kKrlFRL>

SANDOVAL, J. (2017). «DECENT lanzó su plataforma blockchain para distribución de contenido audiovisual». Criptonoticias.com [en línea]. [Consulta: 3 de julio del 2017] <https://criptonoticias.com/aplicaciones/decent-lanzo-plataformablockchain-distribucion-contenido-audiovisual/\#axzz4lkKrlFRL>

STAHR, D. (2014). «Sledováníobarvených Bitcoinů v transakčním». dspace.cuni.cz [en línea]. [Consulta: 25 de junio del 2017]. <https://dspace.cuni.cz/bitstream/ handle/20.500.11956/68852/BPTX_2013_1_11320_0_348269_0_142918. pdf?sequence $=1>$ 
SWAN, M. (2015). «Blockchain: blueprint for a new economy» [en línea]. S. I.: O'Reilly Media. [Consulta: 20 de mayo del 2017]. ISBN 9781491920473. $<$ http://www.goodreads.com/book/show/24714901-blockchain>

TORMEY, J. (2017). «Blockchain Wallet API V2». Londres: GitHub. 0.26.0 [en línea]. $<$ https://github.com/blockchain/service-my-wallet-v3>

UNIDAD EDITORIAL INFORMACIÓN ECONÓMICA (2017). «Bitcoin, ¿ganará el combate contra la banca española?» Expansión [en línea]. [Consulta: 7 de julio del 2017]. <http://www.expansion.com/promociones/native/2017/04/27/>

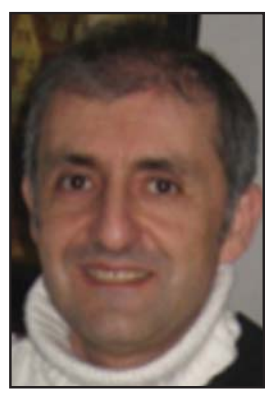

\section{José Miguel Domínguez Jurado josemiguel.dominguez@uca.es Profesor titular de Economía Aplicada (Universidad de Cádiz)}

Doctor en Economía por la Universidad de Cádiz. Máster en Impactos territoriales y ambientales de la globalización por la Universidad Internacional de Andalucía. Licenciado en Ciencias Económicas y Empresariales por la Universidad de Málaga. Es profesor de economía aplicada, e imparte diferentes asignaturas relacionadas con el análisis económico en estudios de grado y de economía regional en máster oficial. Colabora como docente en programas para alumnos de universidades de Estados Unidos, en asignaturas de economía regional y urbana. Ha sido investigador principal de la UCA en el proyecto PRESPO de Interreg de la Unión Europea, y ha participado en otros proyectos de ámbito nacional e internacional.

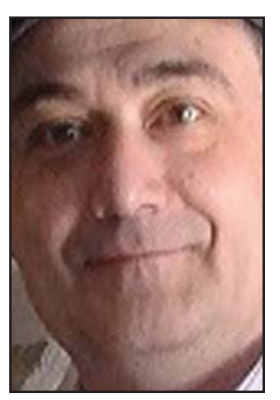

\section{Ricardo García Ruiz} rgarciarui@uoc.edu Profesor colaborador del grado de Información y Documentación (UOC)

Doctor en Economía por la Universidad Internacional de Barcelona. Máster en Sociedad de la información y del conocimiento por la UOC. Ingeniero informático por la UOC. Máster en Técnicas avanzadas de estadística aplicada por la UNED. Experto en métodos avanzados de estadística aplicada por la UNED. Actualmente, colabora como profesor en los estudios del grado de Información y Documentación, en las materias de big data y minería de datos. 
Los textos publicados en esta revista están sujetas -salvo que se insdique el contrario- a una licencia de Reconocimiento 3.0 España de Creative Commons. Podéis copiarlos, distribuirlos, comunicarlos públicamente y hacer obras derivadas siempre que reconozcáis los créditos de las obras (autoría, nombre de la revista, institución editora) de la manera especificada por los autores o por la revista. La licencia completa se puede consultar en http://creativecommons.org/licenses/by/3.0/es/deed.ca.

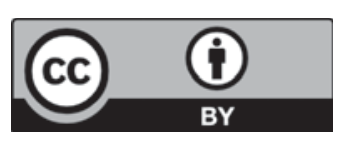

